\title{
An improved DCA and channel modelling algorithm based on carrier priority in LTE/LTE-A
}

\author{
Amirhossein Fereidountabar \\ Department of Electronic Engineering, University of Rome(Tor Vergata), Rome, Italy
}

Email address:

Fereidountabar@ing.uniroma2.it

\section{To cite this article:}

Amirhossein Fereidountabar. An Improved DCA and Channel Modelling Algorithm Based on Carrier Priority in LTE/LTE-A. American Journal of Networks and Communications. Vol. 2, No. 6, 2013, pp. 149-154. doi: 10.11648/j.ajnc.20130206.12

\begin{abstract}
Dynamic Channel Allocation (DCA) technology is an effective ways to solve the contradiction between limited spectrum resources and increasing user demand in TD-SCDMA system. Aiming at the limitation of conventional DCA algorithm, an improved dynamic channel allocation algorithm based on carrier priority is proposed in this paper to solve tidal phenomenon caused by regional traffic migration and shows channel model. The simulation results show that the proposed algorithm can effectively increase resource utilization and improve access success rate with less changing in channel model.
\end{abstract}

Keywords: Channel Modeling, Signal Varying, Dynamic Channel Allocation, Carrier Priority

\section{Introduction}

In TD-SCDMA systems, radio resources include codes, time slots and frequencies [1-2]. There have been a great deal of research on dynamic channel allocation .In[3], traditional switching method, channel reservation algorithm, Queuing priority scheme, Cut off priority scheme and Fractional guard channel scheme were proposed. These methods mainly aimed to optimize for the time slot resources and code resources. The authors of [4] proposed the priority algorithm for services, which use the pilot strength, the moving speed, and other attributes to optimize the queue. However, this algorithm does not consider the carrier properties and carrier priority.

Mobile Phone Service, with obvious flowing distribution and increasing traffic amount, places a huge burden on network, leading to constant "tidal effect" that users tend to converge to different areas at different times, which brings about the migration of traffic and data flow. However, the total traffic and data flow always keep at a basically same level. By reason of sudden and sharp rise in its periodic changes, the traffic may cause frequent service migrations and thus result in low service quality as well as insufficient availability of baseband resource. In this paper, in order to overcome the disadvantage of DCA (dynamic channel allocation) algorithm of current TD-SCDMA system. Utilization of baseband resource, a dynamic channel allocation algorithm has been presented.
This paper is organized as follows. First, the system model is introduced in section 2. Next, the improved DCA algorithm is proposed in section 3. After that, the simulation platform is described briefly, and then the simulation results and some analysis are given in section 4 . Finally, conclusions are drawn in section 5.

\section{System Model}

\subsection{Model for Simulation}

By opposition to statistical channel model approaches which reproduce the global channel behavior, deterministic approaches aim at reproducing real scenarios with physical models representing the interactions between the impinging waves and the local environment. As shown in Fig. 1, a hexagon cellular indicates a cell of the system, where a BS shows a cell's base station in the middle of the cell, of which the position in system can be adjusted by setting its attributes. In the algorithm of this paper, positions of all base stations have been located at centre of the cells, with omnidirectional base station antennas employed. In the model, the hexagon is used to represent the relative location between cells [5]. And, the actual coverage of the base station is determined by system parameters, its transmitting power, cell selection and switching control, etc. The Wrap 
Around technology, for avoiding the "boundary effect", has been used in this paper to build virtual cells by foldingequivalent, so as to simulate the actual interference of the cells that stand at topological edges. Through the foldingequivalent, each cell of the system, either at topology centre or at edge, has been interfered by two layers of adjacent cells [6-7].

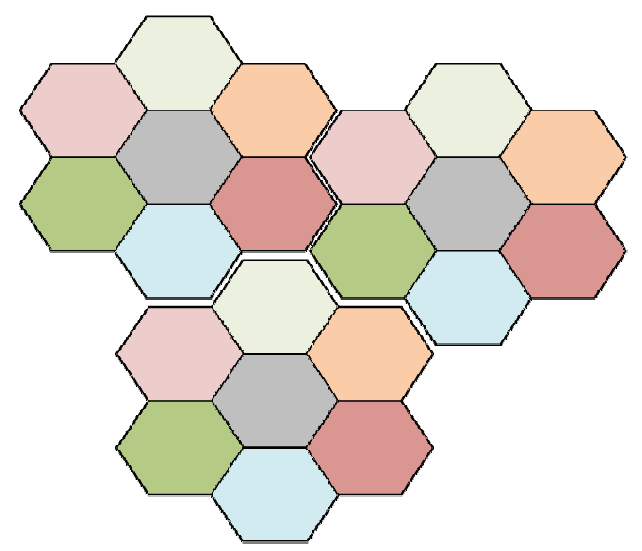

Fig 1. cluster model

\subsection{Node Model}

Station nodes can realize the sending of downlink signals as well as the reception of uplink signals. In radio resource management (RRM), they are responsible for the innerloop power control and fast dynamic channel allocation. UE nodes can achieve the sending of uplink signals and the reception of downlink signals. A UE node has function of mobility and service generation. In RRM, it is mainly to take charge of (inner-loop and outer-loop) power control. UE nodes' measurement to downlink channel and the measurement report are the base of RRM algorithm. RNC nodes are used to bring RRM into effect, including: access control, switching control, load control, DCA and outerloop power control [8-9].

\section{Improvement of DCA Algorithm}

Current DCA algorithm does not concern the shareable attribute of carrier, therefore it may lower the availability of baseband resource, and may give rise to user-adjustment caused by frequently activate the shareable sub-carriers. Therefore, on the basis of concerning the attributes that carriers can be shared and combining cell service, an improved DCA algorithm of dynamically adjusting priority order of carrier has been proposed .Here describes the improved DCA algorithm, as follows:

\subsection{Configure the Carrier Attribute According to It's Mode}

Firstly, we need to take into consideration of the fixed sequencing of BRU (Basic Resource Unit) values, in which the carrier priorities requires to be ordered by operating, managing and maintaining the subsystems, and the ordering method is determined by static setup. When the sequence of priorities has been completed, the specific access sequence of carriers is then needed to be figured out based on the numbers of BRU thresholds. Suppose that carrier priorities are ordered by \{main-carrier, sub-carrier $1, \ldots$, sub-carrier $(\mathrm{N}-1)\}$, then the call request of case that the required BRU numbers is less than the configured BRU thresholds is accessed along with the above order. Otherwise, an opposite order will be employed when the required BRU numbers is more than the configured BRU thresholds.

\subsection{Determination of the Attributes of Main and Sub Carrier}

In a cell, the sub-carrier can be both shared and not shared, while the main carrier can't be shared. Given that the priority of shareable carriers is higher than that of unshareable carriers, so that, when applying the share function, the un-shareable will be allocated with priority to the shareable. By such means, the burden of RNC (Radio Network Controller) caused by frequent user-adjustment, a result of activating the shareable sub-carriers, can be lowered[10].

After the RNC has been allocated with the configured sequence of carrier priorities, we need to check out whether or not there exist activated shareable sub-carriers. If there do exist the activated shareable sub-carriers, then the original carrier priorities should be rearranged according to the principle that the priority of an un-shareable carrier is higher than that of shareable one. Next, the access should be treated by same way[11-12].

\subsection{To Make the Sequence of Carrier Priorities in Accordance with Carrier Attribute Scheme}

Make separate sequences to un-shareable as well as shareable sub-carriers, by following that the priority of a shareable carrier is lower than that of un-shareable one. In this way, carriers of better quality will be selected and allocated in the cells, meanwhile the service quality can be also ensured, and thus improving the availability of baseband resource and access success[13].

As for the sequencing of DCA triggered by cells' shareable sub-carriers, the list of carrier priorities can be updated based on the principle that the priority of a shareable carrier is inferior to an un-shareable one. The arrangement of priorities can be made into a dynamic sequence trough the mean power-load of carriers. In this algorithm, the load of carrier is measured by power. With the periodic test to Node B, the priority of a carrier can be set by calculating its load. And, the setting follows as larger load corresponds to lower priority. Where, uplink measurements can be calculated based on the total power of base station in receiving broadband, or, on the time slot interference on signal code power (ISCP), while downlink measurements are calculated by the total transmitting power of the base station[14-15].

For uplink conditions, the total power of base station in 
receiving broadband can be calculated by (1)

$$
\bar{P}_{j}=\alpha_{P} \cdot \bar{P}_{j, U L}+\left(1-\alpha_{P}\right) \cdot \bar{P}_{j, D L}
$$

Where, $\mathrm{j}$ represents carrier $\mathrm{j}$ of the cell; $N_{j, U L}$ refers to the time slot numbers of carrier $\mathrm{j}$ in uplink service; N0 means the thermal noise power, which is configured through
O\&M; $P_{j, i}$ shows the measured value of time slot $\mathrm{i}$ and carrier j, and; $\bar{P}_{j, U L}$ is the mean of uplink measured value of carrier $\mathrm{j}$.

Equation (2) is used to calculate the ISCP of time slot of uplink conditions:

$$
\begin{aligned}
& \bar{P}_{j, U L}=\left.\frac{1}{N_{j, U L}} \sum_{i=1}^{N_{j, U L}}\left(1-\frac{N_{0}}{P_{j, i}}\right)\right|_{\mathrm{mw}} \text { thus : } \\
& \left.\frac{1}{N_{j, U L}} \sum_{i=1}^{N_{j, U L}}\left(1-10^{\frac{N_{0}-P_{j, i}}{10}}\right)\right|_{\mathrm{dBm}}=\left.\frac{1}{N_{j, U L}} \sum_{i=1}^{N_{j, U L}}\left(1-10^{\frac{N_{0}-\frac{P_{-} P_{j, i}}{10}+112.1}{10}}\right)\right|_{\mathrm{dBm}}
\end{aligned}
$$

Where, ISCP $i, j$ shows the measured value of time slot $i$ and carrier $\mathrm{j}$; ISCP max is the maximum interference value of time slot, which is configured by O\&M.

$$
\begin{aligned}
& \bar{P}_{j, U L}=\left.\frac{1}{N_{j, U L}} \sum_{i=1}^{N_{j, U L}}\left(\frac{I S C P_{j, i}}{I S C P_{\max }}\right)\right|_{\mathrm{mw}} \text { thus : } \\
& \left.\frac{1}{N_{j, U L}} \sum_{i=1}^{N_{j, U L}}\left(10 \frac{I S C P_{j, i}-I S C P_{\max }}{10}\right)\right|_{\mathrm{dBm}}=\left.\frac{1}{N_{j, U L}} \sum_{i=1}^{N_{j, U L}}\left(10 \frac{\frac{P_{-} I S C P_{j, i}}{2}-120.5-I S C P_{\max }}{10}\right)\right|_{\mathrm{dBm}}
\end{aligned}
$$

For downlink conditions, the power of carrier transmitted form base station can be addressed by (3):
Where, $N_{j, D L}$ represents the time slot numbers of carrier $\mathrm{j}$ in downlink service; $\bar{P}_{j, D L}$ points to the statistical mean value of carrier $\mathrm{j}$ in downlink service. For HSDPA carrier, the downlink measurement is made based on NONHSDPA's downlink transmitting carrier power instead of BS transmitting carrier power.Fig. 2 shows the Multi path power in LTE channel.

Finally, use (4) to calculate the average power load of the weighted uplink and downlink carriers.

$\bar{P}_{j, D L}=\frac{1}{N_{j, D L}} \sum_{i=1}^{N_{j, D L}} P_{j, i+N_{j, U L}}=\frac{1}{N_{j, D L}} \sum_{i=1}^{N_{j, D L}} \frac{\mathrm{P}_{-} P_{j, i+N_{j, U L}}}{100}$

Where, $\bar{P}_{j}$ means the average power load of carrier $\mathrm{j}$; $\alpha_{P}$ configured by O\&M, is the weighting factor $\left(\alpha_{P} \in\{0 \ldots 1\}\right)$ of power measurement values. After the average power load has been obtained form (4), the priorities of carriers can be arranged. Now, make a judgment that whether there are activated shareable subcarriers. If there do exist the activated shareable subcarriers, then the original carrier priorities should be rearranged according to the principle that the priority of an un-shareable carrier is higher than that of shareable one[1618]. Fig. 3 shows the process of the priority sequence of carriers. 


\section{Simulation and Results Analysis}

\subsection{Simulation Parameters Setting}

Based on above analysis and description of the algorithm, the simulating tool is thus to be used to build a TDSCDMA system simulation platform for testing the performance of the improved algorithm. A model combined with voice and data service has been employed in simulation. In this model we considered sub-frame cycle is 5ms. Given that call delivery obeys Poisson's distribution, with the average value can be assigned to be $60,120,180$, 240 and 300 calls $/ \mathrm{h}$; and the call lasting time follows negative exponential distribution. Specific simulating parameters refer to table 1 :

Table 1. Simulating Parameters

\begin{tabular}{lc}
\hline Simulating Parameters & Value \\
\hline Sub-frame cycle & $5 \mathrm{~ms}$ \\
Time slot numbers of sub-frame service & 6 \\
Time slot numbers of voice service & Uplink/downlink $=2$ \\
The required BR by voice service & 2 \\
The required BRU by data service & 16 \\
Average lasting time & $120 \mathrm{~s}$ \\
\hline
\end{tabular}

\subsection{Simulation Results Analysis}

In order to analyze the performance of the proposed algorithm we simulate the traditional DCA algorithm and the improved DCA algorithm, then compare them from access success rate, resource utilization rate and average lasting time of data packets.

Fig. 4 and 5 show the simulating results of voice services access success rate and average delays of data packets, respectively, which were obtained from the traditional DCA algorithm and the improved DCA algorithm. It can be concluded from the simulation results that, as the increasing of voice calls and data packet service, the rate of access success of the improved DCA algorithm is higher than that of traditional algorithm, with average delays of data packets lower than traditional one. This is because the sharing of carriers in busy voice and data services can increase the capacity of a single cell, and decrease the network congestion, which gives a higher rate of access success. Fig.6 compares two algorithms.

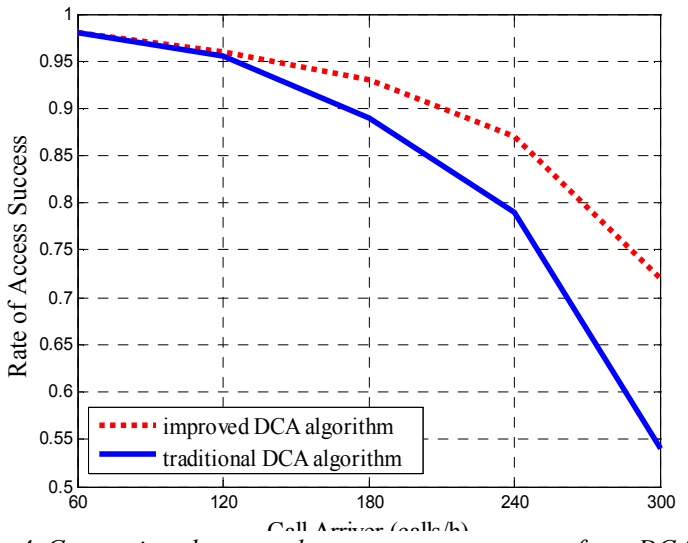

Fig 4. Comparison between the access success rates of two DCA algorithms

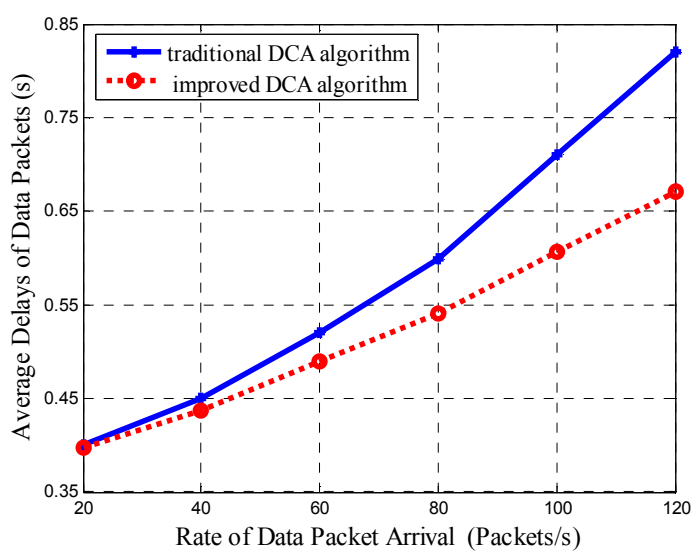

Fig 5. Comparison between the average delays of data packets of two DCA algorithms

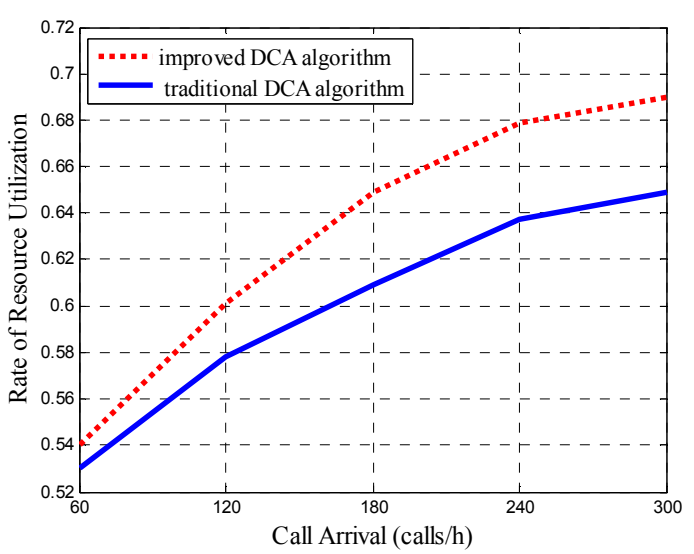

Fig 6. Comparison of resource utilization between two DCA algorithms 


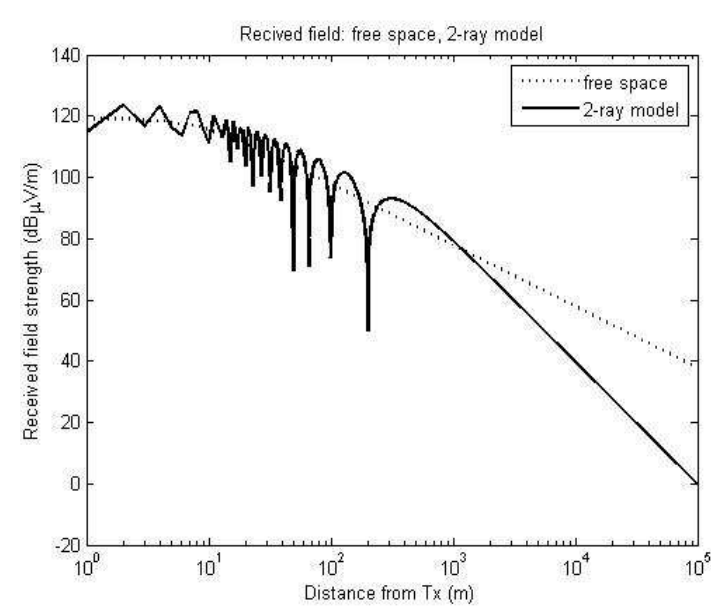

Fig 7. Strength of the received signal

The availabilities of baseband resource of the two algorithms have been compared under the voice service, as shown in Fig. 5. As seen from the results, due to the attribute that carriers have been share, the improved DCA can effectively make use of baseband resource when the rate of call delivery rising up. Fig 7,8 and 9 show strength, magnitude and phase of the received signal respectively.

The speed parameter is here taken into account since it has an important influence on the integration process taking place in the receiver as previously mentioned.

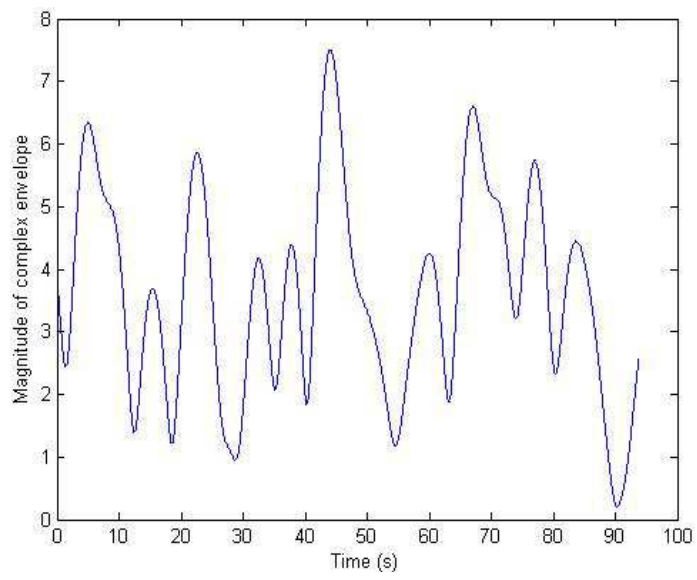

Fig 8. Magnitude of complex envelop

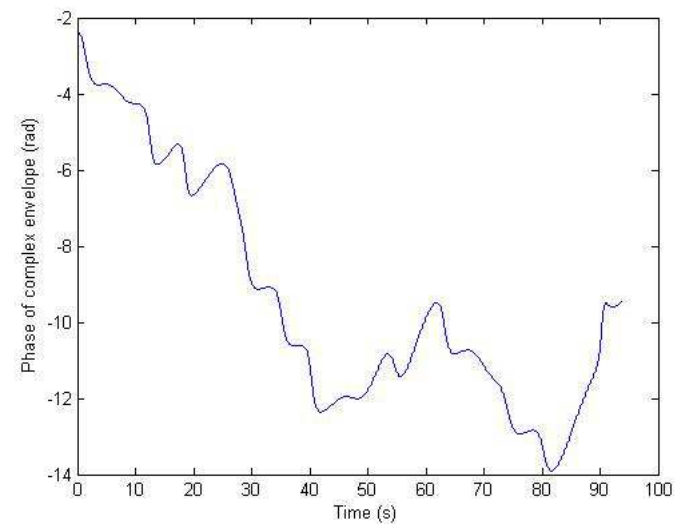

Fig 9. Phase of complex envelop

\section{Conclusions}

In order to solve the "tidal effect" caused by the sudden migration among local areas, an improved DCA algorithm based on the priority of carrier attributes has been presented in this paper. Such an algorithm not only reserves the advantages of traditional DAC algorithm, meanwhile, it can enhance the availability of baseband resource by dynamically adjusting carrier priorities of cell service and stability of the radio channel. The simulation results indicate that the algorithm proposed in this paper can effectively improve the rates of baseband resource utilization as well as access success. In this situation conditions of the channel and improvement of it are shown in time domain.

\section{References}

[1] 3GPP TSG Radio Resource Management Strategies, TR 25.922 ( release5 ), 2002

[2] PEI M G, WANG W B. "TD-SCDMA Mobile Communication System". The Third Edition, Beijing: China Machine Press, 2009.

[3] Li Shi-He . "TD-SCDMA 3G Mobile Communication Standard".Post \& Telecom Press, Beijing, 2003.10.

[4] Hyang Duck Cho, Jae Kyun Park, Wadin KO, Keum sang Lim, Wooshik Kim. "A Study on the MCHO Method in Hard Handover and Soft Handover between WLAN and CDMA”. IEEE,2005:391-392.

[5] Shuhui Liu ; Yongyu Chang ; Ruiming Yang ; Dacheng Yang. "Efficient Distributed Dynamic Resource Allocation for LTE Systems" Vehicular Technology Conference (VTC Fall), 2011 IEEE

[6] Joung Woong Lee. "Advanced Semisoft Handoff Method of Cellular IP Access Networks". Proceedings of the 18th International Conference on Advanced Information Networking and Application (AINA'04), 2004:1.

[7] Kaijie Zhou,"Dynamic resource allocation for machine-type communications in LTE/LTE-A with contention-based access"Wireless Communications and Networking Conference (WCNC), 2013 IEEE.

[8] Qing-An Zeng,Dharma P. Agrawal. "Modeling and Efficient Handling of Handoffs in Integrated Wireless Mobile Networks". IEEE Transactions on Vehicular Technology,2002, 51(6):1469-1478

[9] Wei-Cheng Xiao, Shih-Hsuan Tang, Ling-Jyh Chen, ChengFu Chou. "A Novel Seamless Vertical Hand off Solution". IEEE,2007:1213-1214

[10] Abrardo,A.;Belleschi,M. ;Detti,P .;Moretti,M., “Dynamic resource allocation for machine-type communications in LTE/LTE-A with contention-based access" Wireless Communications, IEEE Transactions on (Volume:11, Issue: $1)$.

[11] Guo Lingyan. "The Influence and Control of Baton Handover in TD-SCDMA ". Guangdong: Sun Yat-Sen University, 2005. 
[12] L.Cao, and C. Comaniciu, "Adaptive channel allocation spectrum etiquette for cognitive radio networks," Mobile Networks and Applications, vol. 11, pp. 779-797, Dec. 2006.

[13] K. Haneda, J. Poutanen, F. Tuvfesson, L. Liu, V. Kolmonen, P. Vainikainen, and C. Oestges, "Development of multi-link geometry-based stochastic channel models," in Antennas and Propagation Conference (LAPC), 2011 Loughborough, Nov. 2011, pp. 1-7.

[14] X.Zhu, L.Shen, T.S.P.Yum, "Analysis of cognitive radio spectrum access with optimum channel reservation," IEEE communication letters vol. 11, no. 4, pp 304-306, April 2007.

[15] J. Poutanen, K. Haneda, V.-M. Kolmonen, J. Salmi, and P. Vainikainen, "Analysis of correlated shadow fading in duallink indoor radio wave propagation," Antennas and Wireless Propagation Letters, IEEE,vol. 8, pp. 1190-1193, 2009.
[16] T. Kurner, "The role of propagation models for the development and deployment of future wireless communication systems," in Electromagnetics in Advanced Applications, 2007. ICEAA 2007. International Conference on, Sept. 2005, 4 pages (electronically).

[17] S.Tang,Brain L.Mark an analytical performance of Opportunistic Spectrum Access in a Military Environment, "in Proc of Wireless Communication and Networking Conference, pp 2681 - 2686, April 2008.

[18] Garcia, F.P. ; Comput. Sci. Dept., Univ. of Fortaleza ; Brayner, A.R. ; Silva, R.J. ; Oliveira, W "Mobile Objects: A Strategy for Supporting Dynamic Channel Allocation in Cellular Networks",IEEE International Conference on Wireless and Mobile Computing, Networking and Communications, 2006. 